\title{
HOMENAJE DE LA CORTE INTERAMERICANA DE DERECHOS HUMANOS AL JUEZ HÉGTOR FIX-ZAMUDIO CON MOTIVO DE SUS 90 AÑOS DE VIDA*
}

\author{
Eduardo FERRER MAC-GREGOR**
}

Señor director de nuestro Instituto,

Señores presidente y ex presidentes de la Corte Interamericana de Derechos Humanos,

Señor ex presidente de la Comisión Interamericana de Derechos Humanos,

Queridas y queridos colegas investigadores y universitarios,

Distinguidas y distinguidos invitados de honor:

\section{INTRODUCCIÓN}

Hoy es un día de fiesta en el que la Corte Interamericana de Derechos Humanos se une al concierto de homenajes que a lo largo de esta semana se ha brindado a un jurista, a un maestro, a un universitario ejemplar, a un

* Palabras pronunciadas en el Homenaje de la Corte Interamericana de Derechos Humanos al Juez Héctor Fix-Zamudio con motivo de sus 90 años de vida, celebrado por el Instituto de Investigaciones Jurídicas de la UNAM y la Corte Interamericana de Derechos Humanos el 5 de septiembre de 2014, en el Aula de Seminarios "Guillermo Floris Margadant" de dicho Instituto; con la participación de Héctor Fix Fierro (director del IIJ), Humberto Sierra Porto (presidente de la Corte IDH), Sergio García Ramírez (ex presidente de la Corte IDH), José de Jesús Orozco Henríquez (ex presidente de la Comisión IDH), Eduardo Ferrer Mac-Gregor (juez de la Corte IDH) y el hoy homenajeado (ex presidente de la Corte IDH).

** Investigador en el Instituto de Investigaciones Jurídicas de la UNAM y juez de la Corte Interamericana de Derechos Humanos.

1 No es la primera vez que la Corte Interamericana realiza un homenaje al doctor Fix-Zamudio, véase Liber Amicorum Héctor Fix-Zamudio, San José, Corte Interamericana de Derechos Humanos, 1998, II vols.; esta obra se realizó en ocasión de su conclusión como juez interamericano.

2 Los homenajes se realizaron entre el 1o. y el 5 de septiembre de 2014 por diversas instituciones donde ha dejado huella el ilustre homenajeado, como la Suprema Corte de Justicia de la Nación, El Colegio Nacional, el Instituto de Investigaciones Jurídicas de la UNAM y la Corte Interamericana de Derechos Humanos.

Boletín Mexicano de Derecho Comparado nueva serie, año XLVIII, núm. 143, mayo-agosto de 2015, pp. 873-878

D. R. C 2015. UNAM, Instituto de Investigaciones Jurídicas. 
escritor prolífico, a un científico, a un juez, a un ser humano excepcional, a nuestro querido maestro, maestro de todos, al doctor Héctor Fix-Zamudio. Es de todos conocidos que el maestro Fix en más de una ocasión declinó el alto honor de convertirse en ministro de la Suprema Corte de Justicia de la Nación, optando por una vida dedicada a la investigación y al magisterio. En esa ruta y decisión vocacional, el destino unió al maestro Fix y a la Corte Interamericana, toda vez que el cargo de juez interamericano no es de dedicación exclusiva, lo que permitió compatibilizar su dedicación vocacional a la investigación y docencia, con la labor de impartir justicia en materia de derechos humanos.

Con el profundo cariño de un discípulo y en ocasión de sus noventa años de fructífera existencia, destacaré algunos destellos de la grandeza del juez Héctor Fix-Zamudio.

\section{EL PASO DEL MAESTRO FIX-ZAMUDIO \\ POR LA GORTE INTERAMERICANA: ESTADÍSTICAS}

El paso del maestro Fix en el sistema interamericano de derechos humanos fue imprescindible para lo que hoy forma parte del corpus juris del derecho internacional de los derechos humanos. Inició funciones como juez de la Corte Interamericana el 1o. de enero de 1986, siendo el noveno juez en formar parte del Tribunal Interamericano y su séptimo presidente, durante dos periodos, comprendidos entre 1990 y 1993, y 1995 y 1997, cumpliendo su encargo el 31 de diciembre de 1997. Esto significa que durante sus doce años de encargo (1986-1997), más de la mitad del tiempo presidió dicho tribunal internacional. Lo anterior no es un dato menor, ya que implicó que en cuatro ocasiones distintas sus colegas lo eligieran para presidir la Corte Interamericana, lo que en sí mismo refleja su liderazgo y autoridad moral.

Como juez de la Corte Interamericana conoció de 18 casos contenciosos en sus diversas etapas. Destaca su participación en los primeros casos resueltos por el Tribunal, los casos Velázquez Rodríguez, Fairén Garbi y Solis Corrales y Godinez Cruz, conocidos como los casos hondureños sobre desaparición forzada. Su participación en casos de desaparición forzada, ejecuciones extrajudiciales, tortura, entre otros, fueron de vital importancia para cimentar lo que hoy es el Sistema Interamericano y cuyos pre- 
Este revista forma parte del acervo de la Biblioteca Jurídica Virtual del Instituto de Investigaciones Jurídicas de la UNAM

cedentes se siguen invocando en la actualidad por el Tribunal Interamericano.

El juez Fix-Zamudio también participó en la resolución de 46 medidas provisionales de las cuales cinco fueron adoptadas durante su primer periodo como presidente de la Corte y 23 durante su segundo periodo en la presidencia. Estas medidas provisionales se dictaron para la protección de testigos de casos de desaparición forzada de personas, la protección de defensores de derechos humanos, la protección de personas privadas de la libertad y de menores, entre otras.

Asimismo, participó en 10 opiniones consultivas entre 1986 y 1997, destacando la opinión consultiva número 6 respecto a la expresión "Leyes" en el artículo 30 de la Convención Americana sobre Derechos Humanos; la opinión consultiva número 7, respecto a la exigibilidad del Derecho de Rectificación o Respuesta; la opinión consultiva número 8, respecto a El Habeas Corpus Bajo Suspensión de Garantías y la opinión consultiva número 9, sobre Garantías Judiciales en Estados de Emergencia, así como la opinión consultiva número 14, respecto a la Responsabilidad Internacional por Expedición y Aplicación de Leyes Violatorias de la Convención, por sólo mencionar algunas de las más relevantes.

\section{EL MAESTRO FIX-ZAMUDIO GOMO PILAR EN LA CONSTRUGCIÓN DEL SISTEMA INTERAMERICANO}

El legado del juez Fix-Zamudio difícilmente podría ser medido en toda su dimensión sólo considerando la estadística que acabo de mencionar. En esta valoración se debe tener en cuenta que las decisiones en las que participó tanto en su calidad de juez y en su momento como presidente del Tribunal, constituye en cierta medida el pilar sobre el cual descansa la actual labor jurisdiccional de la Corte Interamericana.

De manera destacada debo mencionar la sentencia del caso Velásquez Rodríguez, el primer caso contencioso resuelto en cuando al fondo por la Corte Interamericana en 1988 y que con el paso de los años se ha convertido en la sentencia más citada por el propio tribunal y de las más citadas por otros tribunales internacionales como el Tribunal Europeo de Derechos Humanos. Este caso, al igual que los otros casos hondureños sobre desaparición forzada de personas, fueron los primeros de su tipo sometidos a un tribunal internacional y la Corte Interamericana tuvo que crear 
su propio camino para resolver este tipo de controversias estableciendo las bases del funcionamiento del sistema interamericano tanto a nivel procesal como sustantivo, si se tiene en consideración que la Convención Interamericana sobre Desapariciones Forzadas fue adoptada hasta 1994, entrando en vigor en 1996; es decir, la jurisprudencia interamericana contribuyó para la posterior adopción del tratado internacional sobre la materia en el ámbito interamericano.

La labor del maestro Fix es aún más loable si consideramos que en esos primeros años el entorno en el cual la Corte Interamericana daba sus primeros pasos era significativamente diferente a la actual. En esos años en varios países del continente aún existían regímenes militares y dictaduras civiles, lo que muchos académicos de la época veían con exceptisismo las labores de dicho tribunal.

Como ha mencionado el también ex presidente de la Corte Interamericana, Sergio García Ramírez, el maestro Fix fue clave en la consolidación de la Corte en una etapa especialmente importante y difícil: los años inmediatos posteriores a la fundación, durante los que el Tribunal debió establecer su perfil, llevar adelante sus primeros y fundamentales desarrollos jurisprudenciales y acreditar su valor y eficacia antes las diversas instancias del sistema interamericano y ante un amplio conjunto de observadores externos. ${ }^{1}$

El maestro Fix-Zamudo, asimismo, es recordado por su brillante participación como presidente del Tribunal. Desde esa posición encausó importantes debates jurídicos en un ambiente de respeto y cordialidad, el cual es aún recordado por los jueces del tribunal de esa época y por el personal de la Corte que lo llegó a conocer.

Incluso se debe a sus gestiones como presidente el lograr que en 1993 el actual edificio sede de la Corte fuera propio, lo que ha permitido ampliar sus instalaciones, teniendo hoy dignas salas para celebrar las audiencias públicas y realizar las deliberaciones privadas de los jueces. Así, la modesta cazona ubicada a 100 metros sur de la pastelería Spoon de los

1 Cfr. García Ramírez, Sergio, "Héctor Fix-Zamudio: la defensa de los derechos humanos. Reflexiones sobre la Corte Interamericana”, en Ferrer Mac-Gregor, Eduardo y Zaldívar Lelo de Larrea, Arturo (coords.), La ciencia del derecho procesal constitucional. Estudios en homenaje a Héctor Fix-Zamudio en sus cincuenta años como investigador del derecho, México, Instituto Mexicano de Derecho Procesal Constitucional-Marcial PonsUNAM, Instituto de Investigaciones Jurídicas, agosto de 2008, t. IX, pp. 447-452.

D. R. (C) 2015. UNAM, Instituto de Investigaciones Jurídicas, Boletín Mexicano de Derecho Comparado, núm. 143, pp. 873-878 
Este revista forma parte del acervo de la Biblioteca Jurídica Virtual del Instituto de Investigaciones Jurídicas de la UNAM

Yoses ahora es propia y tiene una apariencia mucho más de tribunal, aunque sigue conservando la misma dirección.

\section{EL MAESTRO FIX-ZAMUdIO: \\ UN "PUENTE VIVO" ENTRE EL DERECHO CONSTITUCIONAL E INTERNAGIONAL DE LOS DERECHOS HUMANOS}

Si bien Héctor Fix-Zamudio ha sido un juez que marcó época en la historia de la Corte, también es cierto que fue un visionario y un jurista muy adelantado a su tiempo. En la época en la que el hoy investigador emérito de nuestro Instituto de Investigaciones Jurídicas participó como juez de la Corte Interamericana, aún se veía lejos el día en que dicho tribunal o algún tribunal internacional en las Américas pudiera tener algún tipo de incidencia en nuestro derecho constitucional y en particular en la forma en la que concebíamos nuestro sistema de derechos fundado en las antiguas "garantías individuales". Eventualmente México aceptó la competencia contenciosa de la Corte Interamericana, casi un año después de que el maestro Fix dejara su cargo como juez en diciembre de 1998. Y esto también se debe a la intercesión que el juez Fix-Zamudio realizara con el entonces presidente de México, el doctor Ernesto Zedillo Ponce de León.

En relativamente poco tiempo, el debate nacional en torno a los derechos humanos tuvo un cambio paradigmático, un cambio de rumbo que nos obliga hoy más que nunca a estudiar la jurisprudencia interamericana en la que el maestro Fix-Zamudio ha sido uno de sus más ilustres artífices, tanto en su construcción como en su consolidación. Por ello, no es exagerado decir que el maestro Fix es en verdad un "puente vivo" entre el derecho nacional e internacional de los derechos humanos y que gran parte de la transformación jurídica que se vive en México nunca hubiese sido posible sin su trascendental labor como juez y académico de los derechos humanos.

Los criterios que fueron desarrollados desde la más temprana jurisprudencia en la que participó el juez Héctor Fix-Zamudio hoy en día siguen siendo fundamentales, pues son los casos paradigmáticos sobre los cuales el Sistema Interamericano se construyó para la protección de los derechos humanos en el continente. 
Este revista forma parte del acervo de la Biblioteca Jurídica Virtual del Instituto de Investigaciones Jurídicas de la UNAM

\section{EL COMPROMISO DE SER JUEZ DE LA CORTE \\ INTERAMERICANA DE CARA AL LEGADO \\ DEL MAESTRO FIX-ZAMUDIO}

La decisión y firmeza vocacional del maestro Fix ha sido un ejemplo a seguir por todos los que hemos tenido el honor de ser sus discípulos. Ahora más que nunca le puedo decir, querido maestro, con agradecimiento y humildad, que su sabiduría y entrega durante sus brillantes años en la Corte Interamericana constituye un ejemplo de vida y un estándar muy alto de lo que debe ser un juez interamericano. Es por ello que su legado nos debe conminar a tomar conciencia del largo camino de esfuerzo que se ha tenido que recorrer para que el sistema interamericano sea lo que hoy es y de lo que tenemos que seguir trabajando para que éste siga siendo un faro que guíe el pleno respeto y garantía de los derechos humanos en el continente.

Quiero terminar con las palabras que el ex presidente de la Corte Interamericana, Hernán Salgado Pesantes, escribiera con motivo de la presentación del Liber Amicorum Héctor Fix-Zamudio, publicado por la propia Corte en 1998: ${ }^{2}$

No estaría completa esta breve semblanza si no me refiriera a la gran modestia y a esa enorme sencillez que aparecen como rasgos distintivos de su personalidad y que son signos inequívocos de una elevada estatura moral y de una sabiduría que ha alcanzado un nivel superior de madurez.

Igualmente no podría dejar de mencionar a la esposa del ilustre homenajeado, doña María Cristina, que ha sido el centro de gravitación familiar y la copartícipe espiritual en la obra y actividad del maestro.

Hoy, querido maestro Fix, firmo esta sentencia adoptada por unanimidad, sin voto particular o concurrente.

Enhorabuena, feliz cumpleaños y muchas gracias por todo lo que nos sigue regalando.

2 Salgado Pesantes, Hernán, "Semblanza de Héctor Fix-Zamudio", Liber Amicorum Héctor Fix-Zamudio, San José, Corte Interamericana de Derechos Humanos, 1998, vol. I, p. XLVIII.

D. R. (C) 2015. UNAM, Instituto de Investigaciones Jurídicas, Boletín Mexicano de Derecho Comparado, núm. 143, pp. 873-878 